\title{
INVESTIGATION OF THE COMBUSTION STABILITY OF METHANE-AIR MIXTURE IN RECUPERATIVE BURNERS OF DIFFERENT GEOMETRIES
}

\author{
Alexey Krainov ${ }^{1}$, Leonid Minkov ${ }^{1}$, and Ksenia Moiseeva ${ }^{1, *}$ \\ ${ }^{1}$ National Research Tomsk State University, 634050 Tomsk, Russia
}

\begin{abstract}
The results of numerical investigations of 5.5\% methane-air combustion stability in heat recuperative burners (counter flow burner, Ushape burner and Swiss-roll burner) are presented in this paper. The investigation is carried out with the use of commercial CFD package Ansys-Fluent. The boundaries of combustion stability of 5.5\% methane-air mixture depending on the gas flow rate at the inlet of the burners are determined.
\end{abstract}

The problem of stable combustion of lean gas mixture is of practical importance at the designing energy-efficient and environmentally-friendly burners. The recuperative heat transfer mechanism can be used to initiate and sustain a stable combustion of lean gas mixture [1]. Combustion stability in the recuperative burners is provided by preheating the inflowing cold gas mixture due to heat transfer through the internal partition or through the wall of burner. Among heat recovery devices the counter flow burners [1, 2], U-shaped burner [1,3] and Swiss-roll burner [1] can be distinguished. A configuration of burner has significant influence on the combustion stability of gas. The stable combustion of lean methane-air mixture in the burners of equal channel length but different configurations is achieved under the different conditions.

The investigation results of combustion stability of 5.5\% methane-air mixture in the three recuperative heat exchangers above mentioned are represented in this work. Physical and mathematical formulations of the problem are based on the works $[2,3]$ with taking into account the variety of burner's geometry. The investigation is carried out by means of the commercial code Ansys-Fluent. The aim of this work is to determine the boundaries of combustion stability of 5.5\% methane-air mixture depending on the gas flow rate at the inlet of the burners. The schemes of flow in the counter flow burner, U-shaped burner, Swiss-roll burner are depicted in figs. 1-3 correspondingly. General scheme of the gas flow can be described as follows. Cold methane-air mixture having a velocity $u_{v h}$, a temperature $T_{v h}$, a concentration of fuel species $Y_{v h, C H 4}$ is fed in the inlet channel, the cross-sectional size of which $d$ is greater than a thickness of the internal channel walls.

Corresponding author: Moiseeva_KM@t-sk.ru 
The simplest scheme of the gas flow is that corresponding to the counter flow burner, fig.1. Gas is supposed to be fed with the same flow rate in the two parallel channels connected with a partition wall. The gas flow direction in one channel of the counter flow burner is opposite to that in another channel. In the U-shaped burner the mixture flow passes the inlet channel up to the burner elbow after that it changes the flow direction and flows out through the outlet channel, fig.2. In the Swiss-roll burner having a twisted configuration the mixture flow passes successively through several channels and elbows as shown in fig. 3

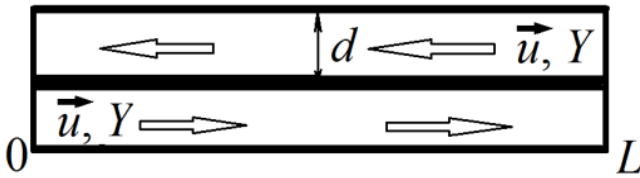

Fig. 1. The counter-flow burner.
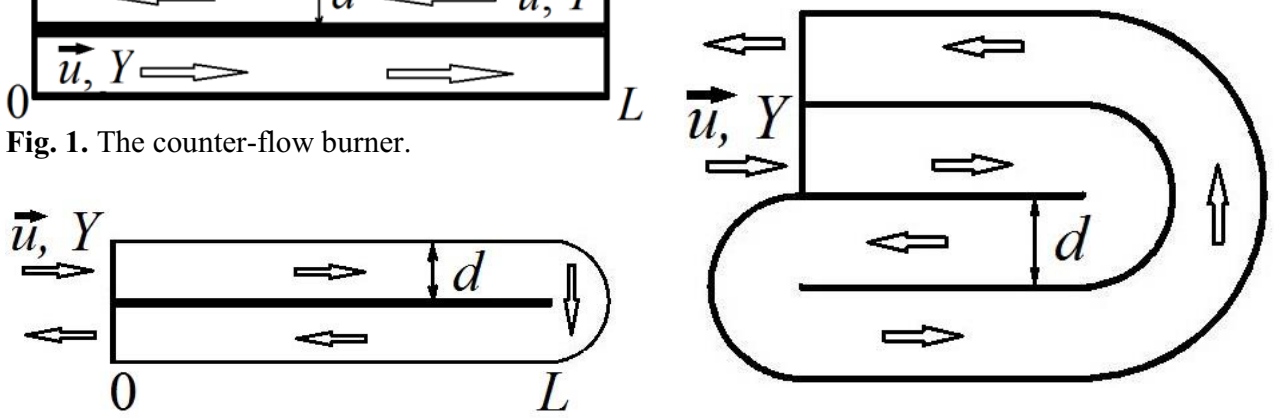

Fig. 2. The U-shape burner.

Fig. 3. The Swiss-roll burner.

The internal walls of a burner are supposed to be uniformly preheated to high temperature at the initial time. The external walls are thermal insulated. A perfect contact between internal walls and gas is supposed to take place.

In formulating the mathematical problem the following assumptions are made: the laminar flow of an incompressible viscous gas is considered; D diffusion coefficient, and $\lambda$ gas thermal conductivity, as well as density and gas flow rate - they all depend on the temperature; the chemical process is supposed to be determined by a one-stage irreversible chemical reaction depending on the temperature according to the Arrhenius law.

The mathematical formulation of the problem is as follows.

The energy equation for the mixture is

$$
\frac{\partial}{\partial t}(\rho E)+\nabla \cdot(\vec{u}(\rho E+p))=\nabla \cdot(\lambda \nabla T)-\sum_{j=1}^{5} h_{j}^{0} R_{j}, x, y \in \text { I, II, III. }
$$

The energy equation for the dividing wall is

$$
\frac{\partial}{\partial t}\left(\rho_{1} E_{1}\right)=\lambda_{1} \nabla^{2} T_{1}, x, y \in \mathrm{IV}
$$

The mass-fraction equations for the species is

$$
\frac{\partial\left(\rho Y_{i}\right)}{\partial t}+\nabla \cdot\left(\rho \vec{u} Y_{i}-\tilde{D}_{m} \nabla Y_{i}\right)=R_{i}, i=1 . .4, x, y \in \text { I, II, III. }
$$

The continuity equation for the mixture is

$$
\frac{\partial \rho}{\partial t}+\nabla \cdot(\rho \vec{u})=0 .
$$


The momentum equation for the mixture is

$$
\frac{\partial(\rho \vec{u})}{\partial t}+\nabla \cdot(\rho \vec{u} \vec{u})+\nabla p=\nabla \cdot \boldsymbol{\tau}
$$

The equation of state for the mixture is

$$
p=\rho R T \sum_{j=1}^{5} \frac{Y_{i}}{M_{i}}
$$

Where $\tau=\mu\left(\nabla \vec{u}+\nabla \vec{u}^{T}\right)$ is the stress tensor, $E=\sum_{j=1}^{5} Y_{j} h_{j}+\frac{\vec{u}^{2}}{2}$ is the total energy of the mixture, $h_{j}=c_{j}\left(T-T_{v h}\right)$ is the enthalpy of $j$-species, $h_{j}^{0}-$ is the enthalpy of formation of $j$-species at the temperature of $T_{v h}, E_{1}=c_{1}\left(T_{1}-T_{1, v h}\right)$ is the enthalpy of the dividing wall, $R_{j}$ is the rate of formation of $j$-species.

The coefficient of thermal conductivity and the coefficient of diffusion for the mixture are assumed to be functions of temperature [3]: $\lambda=\lambda_{s t}\left(\frac{T}{T_{v h}}\right)^{S}, \tilde{D}_{m}=D_{s t} \rho_{s t}\left(\frac{T}{T_{v h}}\right)^{s}$. The rate of formation of $i$-species is determined by formula $R_{i}=\frac{v_{i} M_{i}}{v_{C H 4} M_{C H 4}} k_{0} \rho Y_{C H 4} \exp \left(-\frac{E}{R T}\right)$. Where $i=1-C_{4}, i=2-O_{2}, i=3-$ $\mathrm{CO}_{2}, i=4-\mathrm{H}_{2} \mathrm{O}, i=5-\mathrm{N}_{2}$.

The boundary conditions at the inlet of the burner are as follows: the temperature of the mixture $T_{v h}=300 \mathrm{~K}$, the velocity profile is supposed to be uniform and the velocity value $u_{x, v h}$ is set to variable, the mass fraction of species $Y_{v h, \mathrm{CH} 4}=0.035, Y_{v h, \mathrm{O} 2}=0.23, Y_{v h, \mathrm{CO} 2}=0$, $Y_{v h, H 2 O}=0$.

The boundary conditions at the outer wall of the burner are as follows:

non-slip condition is

$$
\vec{u}=0
$$

zero heat flux condition is

$$
\frac{\partial T}{\partial n}=0
$$

zero species flux condition is

$$
\frac{\partial Y_{i}}{\partial n}=0
$$

The boundary conditions at the inner wall of the burner are as follows:

non-slip condition is

$$
\vec{u}=0 \text {, }
$$

zero species flux condition is 


$$
\frac{\partial Y_{i}}{\partial n}=0
$$

perfect thermal contact is

$$
-\lambda_{1} \frac{\partial T_{1}}{\partial n}=-\lambda \frac{\partial T}{\partial n}, T_{1}=T
$$

Free outflow boundary conditions are used at the outlet of the burner.

The calculations were performed at the thermophysical and kinetic parameters of mixture corresponding to those of work [3]. A length and breadth of channels are set equal to $d=6 \cdot 10^{-3} \mathrm{~m}, d_{1}=2 \cdot 10^{-4} \mathrm{~m}, L=5 \cdot 10^{-2} \mathrm{~m}$. Thermophysical parameters of internal walls are set equal to $c_{1}=687 \mathrm{~J} /(\mathrm{kg} \cdot \mathrm{K}), \quad \lambda_{1}=30 \mathrm{~W} /(\mathrm{m} \cdot \mathrm{K}), \quad \rho_{1}=7500 \mathrm{~kg} / \mathrm{m}^{3}$. The initial temperature of internal walls is equal to $1700 \mathrm{~K}$.

The cross component of mixture velocity $u_{y, v h}$ is assumed to be zero, whereas the longitudinal component of mixture velocity $u_{x, v h}$ varies from $0.01 \mathrm{~m} / \mathrm{s}$ to $0.45 \mathrm{~m} / \mathrm{s}$ in order to determine the maximum value of mixture flow rate, at which the stable combustion is possible.

The calculations were performed using SIMPLE method of the second-order accuracy. The time step was chosen equal to $10^{-5} \mathrm{~s}$, the effect of the boundary layer was taken into account by mesh refinement near the wall, and the maximum step in space was $5 \cdot 10^{-6} \mathrm{~m}$. Conservation of mass, momentum and energy balance was controlled at the computations. It was supposed that the solutions converged to the steady-state ones if residuals of equations were less than $10^{-5}$.

The investigation results are represented in fig. 4-6. The figures shows the results obtained for limit values of the ongitudinal component of mixture velocity at the inlet $u_{x, v h}$. Little increasing in the mixture velocity at the inlet of burner leads to the removal of the combustion front the burner. According to results obtained the boundary of stable combustion for $5.5 \%$ methane-air mixture in the case of equal length channel burners of different types depends on the mixture velocity at the inlet of burners. Stable combustion is possible in the counter flow burner when the limit value of mixture velocity at the inlet $u_{x, v h}$ does not exceed $0.145 \mathrm{~m} / \mathrm{s}$. Changing the burner's configuration from the counter flow one to the U-shaped one (fig.5) enhances the range of the mixture velocity at the inlet of burner $u_{x, v h}$ up to $0.21 \mathrm{~m} / \mathrm{s}$. The twisted channel yields the widest range of the mixture combustion stability (fig.6) $u_{x, v h}=0.3 \mathrm{~m} / \mathrm{s}$.

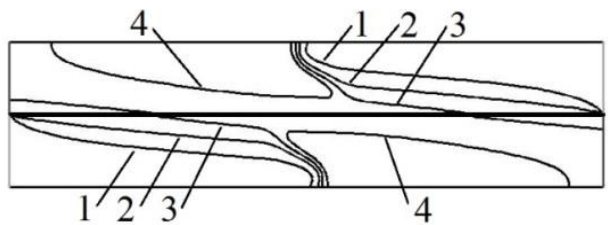

Fig. 4. $U_{x, v h}=0.145 \mathrm{~m} / \mathrm{s} .1-700 K, 2-1100 K$, $3-1500 K, 4-1900 K$.

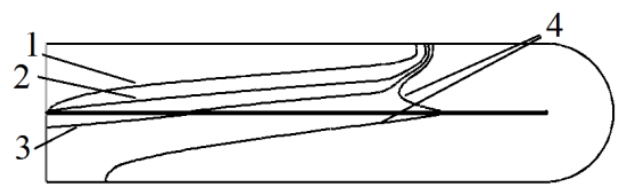

Fig. 5. $U_{x, v h}=0.21 \mathrm{~m} / \mathrm{s} .1-700 K, 2-1100 K, 3-$ $1500 K, 4-1900 K$.

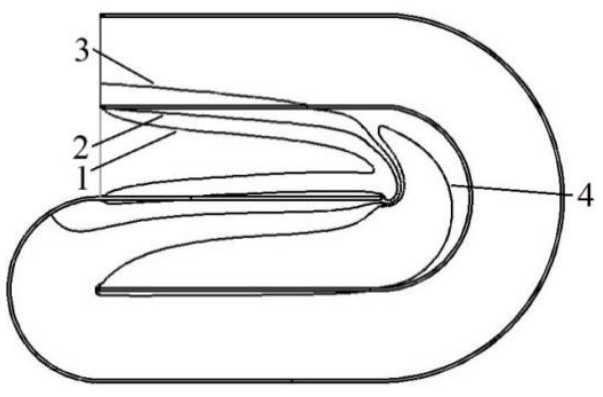

Fig. 6. $U_{x, v h}=0.3 \mathrm{~m} / \mathrm{s} .1-700 K, 2-1100 K$, $3-1500 K, 4-1900 K$. 
The results obtained are in accordance with those of [2-3]. Indeed, the stable mixture combustion in the counter flow burner is possible when the combustion front in the upper channel is situated left of that in the lower channel in the case of right-left feeding for the upper channel and inversely for the lower channel. With that two high temperature zones overlapping each other are developed in the channels. Computations show that the combustion front is established only in the upper part of channel in the case of U-shaped burner that is in accordance with results of [3].

\section{Acknowledgments}

Research is funded by the Russian Science Foundation grant (project no. No. 16-33-60091 mol_a_dk).

\section{References}

1. Y. Ju, K. Maruta, Prog. Energy Combust. Sci. 37 (2011)

2. R. V. Fursenko, S. S. Minaev, Comb., Expl. Shock Waves 41 (2005)

3 A. Yu. Krainov, K. M. Moiseeva, Comb., Expl. Shock Waves 52 (2016)

4. S. Patankar, Numerical heat transfer and fluid flow (Hemisphere Publishing Corporation, New York, 1980) 